\title{
Impact of a Long-Term High Fat Diet on Bone Microarchitecture and Muscle Structure in Adult Male and Female Normal Mice
}

\author{
Weston $\mathrm{He}^{1}$, Trupti Trivedi ${ }^{2}$, Gabriel Pagnotti ${ }^{2}$, Sreemala Murthy ${ }^{2}$, Yun \\ She $^{2}$, Sutha John², Jack Truitt ${ }^{3}$, Khalid S. Mohammad ${ }^{2}$, Theresa A. Guise ${ }^{2}$ \\ ${ }^{1}$ Indiana University School of Medicine Evansville Campus; Evansville, Indiana \\ ${ }^{2}$ Division of Endocrinology, Department of Medicine; Indiana University School of \\ Medicine; Indianapolis, Indiana \\ ${ }^{3}$ Shortridge High School; Indianapolis, Indiana
}

\section{Background and Hypothesis:}

Hyperglycemia is a major source of disease and morbidity among the adult population. Prior studies correlate long-term high fat diet (HFD) mediated hyperglycemia with bone fragility and muscle weakness. Furthermore, the mechanism driving hyperglycemia between sexes are unknown. Our group previously showed that HFDs induced insulin resistance in male mice and glucose intolerance in female mice. This establishes the need to study the impact of long-term HFDs on the bones and muscles using an older cohort of both male and female mice. For that, we hypothesized a long-term HFD mediated hyperglycemia will change bone and muscle structures and impair their functions in adult male and female mice.

\section{Experimental Design or Project Methods:}

22-week C57BI6 mice were fed either a HFD or low fat diet (LFD) for 25 weeks. After euthanasia, bones and muscles were harvested and evaluated using MicroCT, histology, and mechanical testing. Statistical analysis was performed using GraphPad Prism with $p<0.05$ considered significant.

\section{Results:}

MicoCT data saw significant reductions to cortical thickness $(p<0.05)$, bone mineral density $(p<0.001)$, and increases to medullary area $(p<0.05)$ among HFD males and females compared to LFD. HFD-males also experienced significant increase in cortical porosity $(P<0.001)$ whereas no changes were noted in HFDfemales. Trabecular bone volume was relatively unchanged. HFD increased cortical osteoclast surface $(p<0.001)$ for both sexes. Bone histology saw increased marrow adiposity among HFD-females $(p<0.05)$. Muscle histology 
exhibited HFD-related reductions in myofiber diameter $(p<0.001)$ for both sexes. Mechanical testing demonstrated reduced young's modulus $(p<0.05)$ and yield stress $(p<0.05)$ among HFD mice, despite non-significant differences in ultimate strength.

\section{Conclusion and Potential Impact:}

The changes associated with a long-term HFD differed between sexes but still led to functional impairments of bone and muscle for both sexes, emphasizing the importance of looking further into the mechanisms responsible for these changes. This can potentially translate to the clinic in the treatment of musculoskeletal complications associated with HFDs. 